\title{
A note on neighbour-distinguishing regular graphs total-weighting
}

\author{
Jakub Przybyło \\ AGH University of Science and Technology \\ Al. Mickiewicza 30, 30-059 Kraków, Poland \\ przybylo@wms.mat.agh.edu.pl \\ Submitted: May 22, 2007; Accepted: Sep 4, 2008; Published: Sep 15, 2008 \\ Mathematics Subject Classifications: 05C78
}

\begin{abstract}
We investigate the following modification of a problem posed by Karoński, Łuczak and Thomason [J. Combin. Theory, Ser. B 91 (2004) 151-157]. Let us assign positive integers to the edges and vertices of a simple graph $G$. As a result we obtain a vertex-colouring of $G$ by sums of weights assigned to the vertex and its adjacent edges. Can we obtain a proper colouring using only weights 1 and 2 for an arbitrary $G$ ?

We know that the answer is yes if $G$ is a 3-colourable, complete or 4-regular graph. Moreover, it is enough to use weights from 1 to 11, as well as from 1 to $\left\lfloor\frac{\chi(G)}{2}\right\rfloor+1$, for an arbitrary graph $G$. Here we show that weights from 1 to 7 are enough for all regular graphs.
\end{abstract}

Keywords: neighbour-distinguishing total-weighting, regular graph

\section{Introduction}

A $k$-total-weighting of a simple graph $G$ is an assignment of an integer weight, $w(e), w(v) \in$ $\{1, \ldots, k\}$ to each edge $e$ and each vertex $v$ of $G$. A $k$-total-weighting is neighbourdistinguishing (or vertex colouring, see [1,2]) if for every edge $u v, w(u)+\sum_{e \ni u} w(e) \neq$ $w(v)+\sum_{e \ni v} w(e)$. If such a weighting exists, we say that $G$ permits a neighbourdistinguishing $k$-total-weighting.

A similar parameter, but in the case of an edge (not total) weighting, was introduced and studied in [3] by Karoński, Łuczak and Thomason. They asked if each simple connected graph that is not simply a single edge permits a neighbour-distinguishing 3-edgeweighting, and showed that this statement holds for 3-colourable graphs. Then AddarioBerry, Dalal and Reed showed that it is enough to use numbers from 1 to 16 to construct 
a neighbour-distinguishing edge-weighting for an arbitrary graph (not containing a single edge as a component), see [2].

In [4] we conjectured that numbers 1 and 2 in turn are enough to distinguish neighbours of each graph by a total-weighting. We verified this conjecture for some classes of graphs and established the following upper bounds.

Theorem 1 ([4]) All complete, 3-colourable and 4-regular graphs permit neighbour-distinguishing 2-total-weightings.

Theorem 2 ([4]) Each simple graph permits a neighbour-distinguishing 11-total-weighting and a neighbour-distinguishing $\left(\left\lfloor\frac{\chi(G)}{2}\right\rfloor+1\right)$-total-weighting.

Note that a graph permits a neighbour-distinguishing 1-total weighting iff every two neighbours have distinct degrees in this graph. Here we deal then with the most difficult, in a way, case and show that the weights $1, \ldots, 7$ are enough for each regular graph, see Theorem 7 .

\section{Lemmas}

To prove our main result we shall need the following lemmas. Then Corollary 6 will provide us with a construction of a neighbour-distinguishing total-weighting of each regular graph by weights from 1 to 8 , which will be then reduced to 7 by Lemma 4 .

Given a sequence of numbers $\left(a_{1}, \ldots, a_{k}\right)$, we shall call $\left(b_{1}, \ldots, b_{l}\right)$ a block of this sequence iff there exists $0 \leqslant j \leqslant k-l$ such that $b_{i}=a_{j+i}, i=1, \ldots, l$.

Lemma 3 Assume that $s=\left(a_{1}, \ldots, a_{k}\right)$ is a sequence of nonnegative integers such that $a_{1}+\ldots+a_{k} \leqslant k$. Then there is an element $a_{j}=0$ of that sequence such that $a_{j-1}+a_{j+1} \leqslant 3$ (where $\left.a_{0}, a_{k+1}:=0\right)$, unless $s$ consists exclusively of blocks $(1,0,3,0,1)$ and $(1, \ldots, 1)$.

Proof. Let us call the sequences consisting of blocks $(1,0,3,0,1)$ and $(1, \ldots, 1)$ (which may intersect) forbidden. The lemma is obvious for $k \leqslant 3$. It is also easy to verify it for $k=4$, hence let us argue by induction on $k$. Take $k \geqslant 5$ and assume the proposition does not hold for some (not forbidden) sequence $s=\left(a_{1}, \ldots, a_{k}\right)$, hence if $a_{i}=0$, then $a_{i-1}+a_{i+1} \geqslant 4$. If there are two consecutive elements $a_{r}, a_{r+1}$ of $s$ that are either both positive or both equal to 0 , then either the sequence $\left(a_{1}, \ldots, a_{r}\right)$ or $\left(a_{r+1}, \ldots, a_{k}\right)$ is not forbidden and complies with the assumptions of the lemma, hence, by induction, there is an element $a_{j}=0$ such that $a_{j-1}+a_{j+1} \leqslant 3$, a contradiction.

Therefore, we may assume every second element of $s$ is positive and every second one equals 0 . Let $a_{t}$ be the second element that is equal to 0 in the sequence $s$ (hence $t=3$ or 4$)$. By the inequality $a_{i-1}+a_{i+1} \geqslant 4$ for the first of such elements, $a_{1}+\ldots+a_{t} \geqslant 4$. Therefore the sequence $\left(a_{t+1}, \ldots, a_{k}\right)$ complies with the assumptions of the lemma (and is not a forbidden one), hence we again obtain a contradiction by induction. 
Let a $k$-vertex-colouring of $G=(V, E)$ be a proper vertex-colouring $c: V \rightarrow C$ (i.e. $c(u) \neq c(v)$ if $u v \in E)$ by the colours from a colour set $C$ with $|C|=k$. Note that we do not require $c$ to be surjective, hence not all the colours have to be used.

Lemma 4 Let $G$ be a k-regular graph which is neither a complete graph nor an odd cycle. There is a $k$-vertex-colouring with colour classes $V_{1}, \ldots, V_{k}$ such that $d_{V_{i}}(v) \leqslant 3$ for each $v \in V_{i-1}, i=2, \ldots, k$.

Proof. Let $E(U, W)$ denote the set of edges between subsets $U, W$ of the vertex set of $G$. Let also $e(U, W)=|E(U, W)|$. By Brooks' Theorem, there is a $k$-vertex-colouring of $G$. Let us choose such a $k$-vertex-colouring and such an ordering of its colour classes $V_{1}, \ldots, V_{k}$ that minimizes the sum $\sum_{l=2}^{k} e\left(V_{l-1}, V_{l}\right)$. We argue that it complies with our requirements.

Assume it is not so; hence there is $2 \leqslant i \leqslant k$ and $v \in V_{i-1}$ such that $d_{V_{i}}(v) \geqslant 4$. Denote $a_{l}=d_{V_{l}}(v), l=1, \ldots, k\left(a_{0}, a_{k+1}:=0\right)$. Then $a_{i} \geqslant 4\left(a_{i-1}=0\right)$ and, since $G$ is $k$-regular, $a_{1}+\ldots+a_{k}=k$. By Lemma 3, there is $1 \leqslant j \leqslant k$ such that $a_{j}=0$ and $a_{j-1}+a_{j+1} \leqslant 3$, hence $d_{V_{j}}(v)=0$ and we may move $v$ from $V_{i-1}$ to $V_{j}$, and thus at the same time reduce the minimized sum by at least four and add to it at most three (since $v$ has at most three neighbours in $V_{j-1} \cup V_{j+1}$ ), a contradiction.

Led $\delta(G)$ denote the minimal degree of a vertex in a graph $G$. We make use of the following Theorem 5 by Addario-Berry, Dalal and Reed (see [2]) to obtain a similar to their Corollary 6.

Theorem 5 ([2]) Given a graph $G=(V, E)$ and for all $v \in V$, integers $a_{v}^{-}, a_{v}^{+}$such that $a_{v}^{-} \leqslant\left\lfloor\frac{d(v)}{2}\right\rfloor \leqslant a_{v}^{+}<d(v)$, and

$$
a_{v}^{+} \leqslant \min \left(\frac{d(v)+a_{v}^{-}}{2}+1,2 a_{v}^{-}+3\right)
$$

there exists a spanning subgraph $H$ of $G$ such that $d_{H}(v) \in\left\{a_{v}^{-}, a_{v}^{-}+1, a_{v}^{+}, a_{v}^{+}+1\right\}$ for all $v \in V$.

Corollary 6 Given a graph $G=(V, E)$ with $\delta(G)>4$, and for each $v \in V$, integers $a_{v}^{-} \in\left[\left\lfloor\frac{d(v)}{4}\right\rfloor, 2\left\lfloor\frac{d(v)}{4}\right\rfloor\right]$ and $a_{v}^{+}:=a_{v}^{-}+\left\lfloor\frac{d(v)}{4}\right\rfloor+1$, there exists a spanning subgraph $H$ of $G$ such that $d_{H}(v) \in\left\{a_{v}^{-}, a_{v}^{-}+1, a_{v}^{+}, a_{v}^{+}+1\right\}$ for all $v \in V$.

Proof. We have $a_{v}^{-} \leqslant 2\left\lfloor\frac{d(v)}{4}\right\rfloor \leqslant\left\lfloor\frac{d(v)}{2}\right\rfloor,\left\lfloor\frac{d(v)}{2}\right\rfloor \leqslant 2\left\lfloor\frac{d(v)}{4}\right\rfloor+1 \leqslant a_{v}^{+}$and $a_{v}^{+} \leqslant 3\left\lfloor\frac{d(v)}{4}\right\rfloor+1<$ $d(v)$, hence, by Theorem 5, it is enough to prove (1) for all $v \in V$. Note then that $a_{v}^{+}=\frac{a_{v}^{-}}{2}+\frac{a_{v}^{-}}{2}+\left\lfloor\frac{d(v)}{4}\right\rfloor+1 \leqslant \frac{a_{v}^{-}}{2}+\left\lfloor\frac{d(v)}{4}\right\rfloor+\left\lfloor\frac{d(v)}{4}\right\rfloor+1 \leqslant \frac{a_{v}^{-}}{2}+\frac{d(v)}{2}+1$ and $a_{v}^{+}=a_{v}^{-}+\left\lfloor\frac{d(v)}{4}\right\rfloor+1 \leqslant$ $a_{v}^{-}+a_{v}^{-}+1$, thus (1) holds. 


\section{Main Result}

For a given total-weighting $w$ of $G$, let $c_{w}(v):=w(v)+\sum_{e \ni v} w(e)$ (or $c(v)$ for short if the weighting $w$ is obvious), define the resulting colouring for each $v \in V(G)$. We shall call $c(v)$ a colour or a total weight of $v$. Our aim, in fact, is to find such a weighting that this vertex-colouring is proper.

Theorem 7 Each regular graph admits a neighbour-distinguishing 7-total-weighting.

Proof. Let $G$ be a $k$-regular graph. By Theorem 1, we may assume that $G$ is not a complete graph and, by Theorem 2 (and Brooks' Theorem), that $k \geqslant 14$. By Lemma 4 there is a $k$-vertex-colouring with colour classes $V_{1}, \ldots, V_{k}$ such that $d_{V_{4 i}}(v) \leqslant 3$ for each $v \in V_{4(i-1)}, i=2, \ldots,\left\lfloor\frac{k}{4}\right\rfloor$. We shall make use of this fact in the second part of the proof.

Let $s_{i}=k+4\left\lfloor\frac{k}{4}\right\rfloor+4+i$ and $b_{i}=k+8\left\lfloor\frac{k}{4}\right\rfloor+8+i$, and let $L_{i}=\left\{s_{i}, b_{i}\right\}$ be a list of admissible colours (total weights) assigned to the vertex set $V_{i}, i=1, \ldots, k$. In the first part of the proof we construct an 8-total-weighting such that $c_{w}(v) \in L_{i}$ for each $v \in V_{i}, i=1, \ldots, k$. This way, since $s_{1}<\ldots<s_{k}<b_{1}<\ldots<b_{k}$, this weighting will be neighbour-distinguishing. In fact we will use only weights 1 and 5 for the edges. In the second part of the proof we will reduce the weights of some vertices and increase some of the edge weights, so that $w(e) \in\{1,2,5,6\}$ and $1 \leqslant w(v) \leqslant 7$ for all $e \in E$ and $v \in V$, and so that the lists of admissible colours remained the same for all colour classes but those of the form $V_{4 j}, 1 \leqslant j \leqslant\left\lfloor\frac{k}{4}\right\rfloor$. In these classes, we will admit colours in $L_{4 j}^{\prime}=\left\{s_{4 j}-4, s_{4 j}, b_{4 j}-4, b_{4 j}\right\}$ instead of $L_{4 j}, j=1, \ldots\left\lfloor\frac{k}{4}\right\rfloor$. Since $s_{4 j}-4=s_{4(j-1)}$ and $b_{4 j}-4=b_{4(j-1)}$, the total weights of the vertices in $V_{4 j}, j=1, \ldots\left\lfloor\frac{k}{4}\right\rfloor$, will have to be constructed carefully, so that the weighting remains neighbour-distinguishing. Note in particular that $s_{4}-4<s_{1}$ and $s_{k}<b_{4}-4<b_{1}$, hence colouring with $L_{4}^{\prime}$ (instead of $L_{4}$ ) does not produce any new conflicts.

Let us then first weight all the edges of $G$ with 1 and set a temporary weight 0 for each vertex of this graph. This way, each vertex gets a temporary colour $k$. Now for each $v \in V_{4 j+l}$ set $a_{v}^{-}=\left\lfloor\frac{k}{4}\right\rfloor+j$ and $a_{v}^{+}=a_{v}^{-}+\left\lfloor\frac{k}{4}\right\rfloor+1, j=0, \ldots,\left\lfloor\frac{k}{4}\right\rfloor, l=1, \ldots, 4$, (hence $\left.a_{v}^{-} \in\left[\left\lfloor\frac{k}{4}\right\rfloor, 2\left\lfloor\frac{k}{4}\right\rfloor\right]\right)$. Then by Corollary 6 there exists a spanning subgraph $H$ of $G$ such that $d_{H}(v) \in\left\{a_{v}^{-}, a_{v}^{-}+1, a_{v}^{+}, a_{v}^{+}+1\right\}$ for all $v \in V$. Let us then add 4 to the weight of each edge of this subgraph (hence $w(e) \in\{1,5\}$ for $e \in E$ ). Now each vertex $v \in V_{4 j+l}$ has a temporary colour in the set $\left\{k+4\left\lfloor\frac{k}{4}\right\rfloor+4 j, k+4\left\lfloor\frac{k}{4}\right\rfloor+4 j+4, k+8\left\lfloor\frac{k}{4}\right\rfloor+4 j+4, k+\right.$ $\left.8\left\lfloor\frac{k}{4}\right\rfloor+4 j+8\right\}=\left\{s_{4 j+l}-4-l, s_{4 j+l}-l, b_{4 j+l}-4-l, s_{4 j+l}-l\right\}$. Therefore by setting either $w(v)=l+4$ or $l$, we obtain $c(v) \in L_{i}$ and $1 \leqslant w(v) \leqslant 8$ for all $v \in V_{i}, i=1, \ldots, k$. This finishes the first part of the proof.

Note that we may have $w(v)=8$ only for vertices in $V_{4 j}, j=1, \ldots,\left\lfloor\frac{k}{4}\right\rfloor$. We shall reduce these weights in the following manner. Process the vertex sets of the form $V_{4 j}$ one after another in the reversed order, starting from $V_{4\left\lfloor\frac{k}{4}\right\rfloor}$ and ending at $V_{4}$. For a given $V_{4 j}$, process all its vertices in an arbitrary order. We introduce some changes only if $v \in V_{4 j}$ is weighted with 8. Namely, if it has any neighbour in $V_{4(j-1)}$, we choose one such neighbour arbitrarily (call it $u$ ), and reduce the weights of $u$ and $v$ by 1 (it is each time possible since $u$ has at most 3 neighbours in $V_{4 j}$, and had a weight 4 or 8 after the 
first part of the construction), and add 1 to the weight of the edge $u v$ (changing it to 2 or 6), hence the total weights of $v$ and $u$ remain unchanged. On the other hand, if $v$ has no neighbour in $V_{4(j-1)}$ (or $(j=1)$ ), we reduce the weight of $v$ by 4 , hence $c(v) \in L_{4 j}^{\prime}$. Since $v$ has no neighbour in $V_{4(j-1)}$ (for $j>1$ ) and $s_{4}-4<s_{1}, s_{k}<b_{4}-4<b_{1}$, no conflict will appear. After processing all the vertices as described, we therefore obtain a neighbour-distinguishing 7-total-weighting.

\section{References}

[1] L. Addario-Berry, R.E.L. Aldred, K. Dalal, B.A. Reed, Vertex Colouring Edge Partitions, J. Combin. Theory, Ser. B 94 (2) (2005) 237-244.

[2] L. Addario-Berry, K. Dalal, B.A. Reed, Degree constrained subgraphs, Proceedings of GRACO2005, volume 19 of Electron. Notes Discrete Math., Amsterdam (2005), 257-263 (electronic), Elsevier.

[3] M. Karoński, T. Łuczak, A. Thomason, Edge weights and vertex colours, J. Combin. Theory, Ser. B 91 (2004) 151-157.

[4] J. Przybyło, M. Woźniak 1,2 Conjecture, II, Preprint MD 026 (2007), http://www.ii.uj.edu.pl/preMD/index.php. 